DOI 10.4171/JEMS/272

András Némethi

\title{
The Seiberg-Witten invariants of negative definite plumbed 3-manifolds
}

Received March 5, 2010

\begin{abstract}
Assume that $\Gamma$ is a connected negative definite plumbing graph, and that the associated plumbed 3-manifold $M$ is a rational homology sphere. We provide two new combinatorial formulae for the Seiberg-Witten invariant of $M$. The first one is the constant term of a "multivariable Hilbert polynomial', it reflects in a conceptual way the structure of the graph $\Gamma$, and emphasizes the subtle parallelism between these topological invariants and the analytic invariants of normal surface singularities. The second formula realizes the Seiberg-Witten invariant as the normalized Euler characteristic of the lattice cohomology associated with $\Gamma$, supporting the conjectural connections between the Seiberg-Witten Floer homology, or the Heegaard-Floer homology, and the lattice cohomology.
\end{abstract}

Keywords. Normal surface singularities, resolutions of singularities, links of singularities, plumbed 3-manifolds, plumbing graphs, Seiberg-Witten invariants, surgery formulae, periodic constant, Hilbert polynomials, Seiberg-Witten Invariant Conjecture, zeta function, lattice cohomology, Heegaard-Floer homology

\section{Introduction}

Let $\Gamma$ be a connected negative definite plumbing graph with vertices $\mathcal{V}$. We assume that it is a tree, and all the plumbed surfaces have genus zero. Hence, the associated oriented plumbed 3-manifold $M=M(\Gamma)$ is a rational homology sphere. We denote by $\mathfrak{s w}_{\sigma}(M)$ the Seiberg-Witten invariants of $M$ indexed by the $\operatorname{spin}^{c}$-structures $\sigma$ of $M$. Although in recent years several combinatorial formulae were established for them, their computation is still very difficult and involved. E.g., in [Ni04] it is proved that they are equivalent to Turaev's torsion normalized by the Casson-Walker invariant (a result based on the surgery formulas of [MW02]). In terms of $\Gamma$, a combinatorial formula for the Casson-Walker invariant can be deduced from Lescop's book [L96], while Turaev's torsion is determined in [NN02]. Nevertheless, this expression of the torsion is based on a Dedekind-Fourier sum, which, in most of the particular cases, is hard to determine.

For some special graphs, for the computation of the Seiberg-Witten invariant one can use results of the Heegaard-Floer homology too, especially surgery formulae; see e.g.

A. Némethi: Rényi Institute of Mathematics, 1053 Budapest, Reáltanoda u. 13-15, Hungary; e-mail: nemethi@renyi.hu

Mathematics Subject Classification (2010): Primary 32S05, 32S25, 57M27; Secondary 32S45, 32S50, 32C 35, 57R57 
[OSz03b, N05, R04]. Moreover, for arbitrary graphs, [BN10] provides a different type of surgery formula (motivated by singularity theory). In fact, in this note we rely exactly on this surgery formula from [BN10].

Our goal is to provide two new combinatorial formulae for $\mathfrak{s w}_{\sigma}(M)$. One of them uses qualitative properties of the coefficients of a combinatorial zeta function associated with $\Gamma$, the other is the normalized Euler characteristic of the lattice cohomology of $\Gamma$ (introduced in [N08a]). Both formulae reflect in the most conceptual and optimal way the structure of the graph $\Gamma$, and emphasize the subtle parallelism between these topological invariants and the analytic invariants of normal surface singularities. The main aim is to establish the identity (and unity) of these three objects: Seiberg-Witten invariant, periodic constant of the zeta function, and the Euler characteristic of the lattice cohomology.

In order to formulate these correspondences, let us consider the plumbed 4-manifold $\tilde{X}$ associated with $\Gamma$. Its second homology $L$ is freely generated by the 2 -spheres $\left\{E_{v}\right\}_{v \in \mathcal{V}}$, and its second cohomology $L^{\prime}$ by the (anti)dual classes $\left\{E_{v}^{*}\right\}_{v \in \mathcal{V}}$; the intersection form $I=\left(\right.$, ) embeds $L$ into $L^{\prime}$; for details see Subsection 2.1. (Equivalently, $L$ is the combinatorial lattice with intersection form $I$ associated with $\Gamma$, and $L^{\prime}$ is its dual lattice, and both are endowed with their natural bases.) Set $x^{2}:=(x, x)$.

Let $K \in L^{\prime}$ be the canonical class (see 2.1.1), $\widetilde{\sigma}_{c a n}$ the canonical $\operatorname{spin}^{c}$-structure on $\widetilde{X}$ (with $c_{1}\left(\widetilde{\sigma}_{c a n}\right)=-K$ ) and $\sigma_{c a n} \in \operatorname{Spin}^{c}(M)$ its restriction on $M$ (see 3.3.1.

Consider the multi-variable Taylor expansion $Z(\mathbf{t})=\sum p_{l^{\prime}} \mathbf{t}^{l^{\prime}}$ at the origin of

$$
\prod_{v \in \mathcal{V}}\left(1-\mathbf{t}^{E_{v}^{*}}\right)^{\delta_{v}-2},
$$

where for any $l^{\prime}=\sum_{v} l_{v} E_{v} \in L^{\prime}$ we write $\mathbf{t}^{l^{\prime}}=\prod_{v} t_{v}^{l_{v}}$, and $\delta_{v}$ is the valency of $v$. This lives in $\mathbb{Z}\left[\left[L^{\prime}\right]\right]$, the submodule of formal power series $\mathbb{Z}\left[\left[\mathbf{t}^{ \pm 1 / d}\right]\right]$ in variables $\left\{t_{v}^{ \pm 1 / d}\right\}_{v}$, where $d=\operatorname{det}(-I)$. The first identity is the following.

Theorem A. Fix some $l^{\prime} \in L^{\prime}$. Assume that for any $v \in \mathcal{V}$ the $E_{v}^{*}$-coordinate of $l^{\prime}$ is larger than or equal to $-\left(E_{v}^{2}+1\right)$. Then the sum

$$
\sum_{l \in L, l \nsupseteq 0} p_{l^{\prime}+l}
$$

equals a multivariable quadratic function on $l^{\prime}$, namely

$$
-\frac{\left(K+2 l^{\prime}\right)^{2}+|\mathcal{V}|}{8}-\mathfrak{s}_{\left[-l^{\prime}\right]},
$$

where the constant $\mathfrak{s}_{\left[-l^{\prime}\right]}$ depends only on the class $\left[-l^{\prime}\right]$ of $-l^{\prime}$ in $L^{\prime} / L=H^{2}(M, \mathbb{Z})$. Moreover, if $*$ denotes the (torsor) action of $L^{\prime} / L$ on $\operatorname{Spin}^{c}(M)$, one has

$$
\mathfrak{s}_{\left[l^{\prime}\right]}=\mathfrak{s w}_{\left[l^{\prime}\right] * \sigma_{\text {can }}}(M) .
$$

In particular, the normalized Seiberg-Witten invariant appears as the constant term of the 'combinatorial multivariable Hilbert polynomial' (1.0.2). 
For the second identity let us consider the lattice cohomology $\mathbb{H}^{*}(\Gamma)$ associated with $\Gamma$. It depends only on $M$, and it has a natural direct sum decomposition indexed by $\sigma \in$ $\operatorname{Spin}^{c}(M)$, namely $\mathbb{H}^{*}(\Gamma)=\bigoplus_{\sigma} \mathbb{H}^{*}(\Gamma, \sigma)$. Let $\operatorname{eu}\left(\mathbb{H}^{*}(\Gamma, \sigma)\right)$ be the normalized Euler characteristic of the corresponding summand; for more details see 2.3. Then one has

Theorem B. For any $\Gamma$ and $\sigma$ as above

$$
-e u\left(\mathbb{H}^{*}(\Gamma, \sigma)\right)=\mathfrak{s w}_{\sigma}(M(\Gamma)) .
$$

In fact, Theorem A was motivated by a similar formula valid for equivariant geometric genera of normal surface singularities (cf. [N08c]); this is explained in 2.2. The combinatorial quadratic Hilbert-polynomial type behaviour 1.0 .2 is proved in Theorem 3.1.1. It uses essentially the preparatory part of Subsection 2.3. where we review and prove some statements about lattice cohomology, and we identify the two combinatorial objects as

$$
\mathfrak{s}_{\left[-l^{\prime}\right]}=-e u\left(\mathbb{H}^{*}\left(\Gamma,\left[K+2 l^{\prime}\right]\right)\right) .
$$

The second part of Theorem A relies on a surgery formula for the constant term $\mathfrak{s}$, which fits perfectly with the surgery formula proved for the Seiberg-Witten invariant $\mathfrak{s w}$ in [BN10]. This allows us to prove in Subsection 3.3 the identity $\mathfrak{s}=\mathfrak{s w}$ by induction on $|\mathcal{V}|$.

The surgery formula involves in a crucial way the 'periodic constant' of a series introduced in [NO09, O08] (see 3.2.8). In fact, via Theorem A, the Seiberg-Witten invariants can be interpreted as the 'multivariable periodic constants' of the series $Z(\mathbf{t})$.

The series $Z(\mathbf{t})$ was used in several articles studying invariants of surface singularities [CDG04, CDG08, CHR04, N08b, N08c]. Theorem A puts the results of these articles in a new light. Indeed, as a consequence of the present work, the identity of $Z(\mathbf{t})$ with the analytic invariant $\mathcal{P}(\mathbf{t})$ (see 2.2 for its definition), in some articles called the CampilloDelgado-Gusein-Zade type identity, implies automatically the Seiberg-Witten Invariant Conjecture of Nicolaescu and the author (cf. [NN02, N03]). This provides a conceptual understanding of how the Seiberg-Witten invariants appear in a natural way in the world of singularities, and why they can serve as topological candidates for the equivariant geometric genera.

Theorem B follows from Theorem A and 1.0.3. It also has the following interpretation. It is known that the Seiberg-Witten invariant appears as the normalized Euler characteristic of the Heegaard-Floer theory of Ozsváth and Szabó (see [OSz03a, N05, R04]), or of the Seiberg-Witten Floer homology. Theorem B says that the normalized Euler characteristics of these cohomology theories and of the lattice cohomology coincide. This supports the conjecture from [N08a] which predicts a precise correspondence between the corresponding cohomology modules and the normalization terms.

\section{Notation and preliminary results}

\subsection{Surface singularities and their graphs}

Let $(X, o)$ be a complex normal surface singularity whose link $M$ is a rational homology sphere. Let $\pi: \widetilde{X} \rightarrow X$ be a good resolution with dual graph $\Gamma$ whose vertices are 
denoted by $\mathcal{V}$. Hence $\Gamma$ is a tree and all the irreducible exceptional divisors have genus 0 . We will write $s$, or $|\mathcal{V}|$, for the number of vertices.

Set $L:=H_{2}(\widetilde{X}, \mathbb{Z})$. It is freely generated by the classes of the irreducible exceptional curves $\left\{E_{v}\right\}_{v \in \mathcal{V}}$. They will also be identified with the integral cycles supported on $E=$ $\pi^{-1}(o)$. We set $I_{v w}=\left(E_{v}, E_{w}\right)$. The intersection matrix $I=\left\{I_{v w}\right\}$ is negative definite, and any connected plumbing graph with negative definite intersection form appears in this way for some singularity. We write $e_{v}$ for $E_{v}^{2}$.

If $L^{\prime}$ denotes $H^{2}(\widetilde{X}, \mathbb{Z})$, then the intersection form provides an embedding $L \hookrightarrow L^{\prime}$ with factor $H^{2}(\partial \widetilde{X}, \mathbb{Z}) \simeq H_{1}(M, \mathbb{Z}) ;\left[l^{\prime}\right]$ denotes the class of $l^{\prime}$. The form $($, ) extends to $L^{\prime}$ (since $L^{\prime} \subset L \otimes \mathbb{Q}$ ). $L^{\prime}$ is freely generated by the duals $E_{v}^{*}$, where we prefer the convention $\left(E_{v}^{*}, E_{w}\right)=-1$ for $v=w$, and $=0$ otherwise.

The canonical class $K \in L^{\prime}$ is defined by the adjunction formulae

$$
\left(K+E_{v}, E_{v}\right)+2=0 \quad \text { for all } v \in \mathcal{V} \text {. }
$$

For $l_{1}, l_{2} \in L^{\prime}$ one writes $l_{1} \geq l_{2}$ if $l_{1}-l_{2}=\sum r_{v} E_{v}$ with all $r_{v} \in \mathbb{Q}_{\geq 0}$. Denote by $\mathcal{S}^{\prime}$ the Lipman cone $\left\{l^{\prime} \in L^{\prime}:\left(l^{\prime}, E_{v}\right) \leq 0\right.$ for all $\left.v\right\}$. It is generated over $\mathbb{Z}_{\geq 0}$ by the elements $E_{v}^{*}$. Since all the entries of $E_{v}^{*}$ are strictly positive, it follows that for any fixed $a \in L^{\prime}$,

$$
\left\{l^{\prime} \in \mathcal{S}^{\prime}: l^{\prime} \nsupseteq a\right\} \text { is finite. }
$$

\subsection{Motivation of Theorem A: Hilbert series}

One of the strongest analytic invariants of $(X, o)$ is its equivariant divisorial Hilbert series $\mathcal{H}(\mathbf{t})$. This is defined as follows (for more details, see e.g. [N08c, §2 and §3] and [N08b]).

Fix a resolution $\pi$ of $(X, o)$ as in 2.1, let $c:(Y, o) \rightarrow(X, o)$ be the universal abelian cover of $(X, o), \pi_{Y}: \widetilde{Y} \rightarrow Y$ the normalized pullback of $\pi$ by $c$, and $\widetilde{c}: \widetilde{Y} \rightarrow \widetilde{X}$ the morphism which covers $c$. Then $\mathcal{O}_{Y, o}$ inherits the divisorial multi-filtration (cf. [N08b] (4.1.1)]):

$$
\mathcal{F}\left(l^{\prime}\right):=\left\{f \in \mathcal{O}_{Y, o}: \operatorname{div}\left(f \circ \pi_{Y}\right) \geq \widetilde{c}^{*}\left(l^{\prime}\right)\right\} .
$$

Let $\mathfrak{h}\left(l^{\prime}\right)$ be the dimension of the $\left[l^{\prime}\right]$-eigenspace of $\mathcal{O}_{Y, o} / \mathcal{F}\left(l^{\prime}\right)$. Then the equivariant divisorial Hilbert series is

$$
\mathcal{H}(\mathbf{t})=\sum_{l^{\prime}=\sum l_{v} E_{v} \in L^{\prime}} \mathfrak{h}\left(l^{\prime}\right) t_{1}^{l_{1}} \cdots t_{s}^{l_{s}}=\sum_{l^{\prime} \in L^{\prime}} \mathfrak{h}\left(l^{\prime}\right) \mathbf{t}^{l^{\prime}} \in \mathbb{Z}\left[\left[L^{\prime}\right]\right] .
$$

In $\mathcal{H}(\mathbf{t})$ the exponents $l^{\prime}$ of the terms $\mathbf{t}^{l^{\prime}}$ also reflect the $L^{\prime} / L \simeq H_{1}(M, \mathbb{Z})$ eigenspace decomposition. E.g., $\sum_{l \in L} \mathfrak{h}(l) \mathbf{t}^{l}$ corresponds to the $H_{1}(M, \mathbb{Z})$-invariants, hence it is the Hilbert series of $\mathcal{O}_{X, o}$ associated with the $\pi^{-1}(o)$-divisorial multi-filtration (considered and intensively studied e.g. in [CHR04]; see also the citations therein, and [CDG04]).

If $l^{\prime}$ is in the 'special zone' $-K+\mathcal{S}^{\prime}$, then by vanishing (of a first cohomology), and by Riemann-Roch, one finds (see [N08c]) that the expression

$$
\mathfrak{h}\left(l^{\prime}\right)+\frac{\left(K+2 l^{\prime}\right)^{2}+|\mathcal{V}|}{8}
$$


depends only on the class $\left[l^{\prime}\right] \in L^{\prime} / L$ of $l^{\prime}$. In several efforts to connect $\mathcal{H}(\mathbf{t})$ with the topology of the link (i.e. with the combinatorics of the graph $\Gamma$ ), the key bridge is provided by the series (cf. [CDG04, CDG08, N08b, N08c])

$$
\mathcal{P}(\mathbf{t})=-\mathcal{H}(\mathbf{t}) \cdot \prod_{v}\left(1-t_{v}^{-1}\right) \in \mathbb{Z}\left[\left[L^{\prime}\right]\right] .
$$

Moreover, this identity (though it suggests that $\mathcal{P}$ contains less information than $\mathcal{H}$ ) can be 'inverted' (cf. [N08c, (3.2.6)]):

$$
\mathfrak{h}\left(l^{\prime}\right)=\sum_{l \in L, l \nsupseteq 0} \bar{p}_{l^{\prime}+l}, \quad \text { where } \mathcal{P}(\mathbf{t})=\sum_{l^{\prime}} \bar{p}_{l^{\prime}} \mathbf{t}^{l^{\prime}} .
$$

( $\mathcal{P}$ is supported on $\mathcal{S}^{\prime}$, see e.g. [N08c (3.2.2)], hence the sum is finite, cf. 2.1.2.) In particular, by 2.2.1,

$$
\sum_{l \in L, l \geq 0} \bar{p}_{l^{\prime}+l}=- \text { const }_{\left[-l^{\prime}\right]}-\frac{\left(K+2 l^{\prime}\right)^{2}+|\mathcal{V}|}{8}
$$

for any $l^{\prime} \in-K+\mathcal{S}^{\prime}$, where const ${ }_{\left[-l^{\prime}\right]}$ depends only on the class $\left[-l^{\prime}\right]$ of $-l^{\prime}$. The right hand side can be interpreted as a 'multivariable Hilbert polynomial' of degree 2 associated with the series $\mathcal{H}(\mathbf{t})$, or with $\mathcal{P}(\mathbf{t})$.

The point is that $\mathcal{P}(\mathbf{t})$ has a topological candidate, namely $Z(\mathbf{t})$ (for its definition see (1.0.1) from the Introduction), which for several singularities agrees with $\mathcal{P}(\mathbf{t})$ (cf. CDG08, N08b, N08c]). In this way, for such singularities, one gets a topological characterization of the constant terms from 2.2.2. Since these constants (equivariant geometric genera, cf. [N08c]) by the conjectures of [NN02, N03, N07] equal the normalized Seiberg-Witten invariants of the link (for 'nice' analytic structures), one expects that the series $Z(\mathbf{t})$ also admits a multivariable Hilbert polynomial, similar to the right hand side of 2.2.2 with constant terms the normalized Seiberg-Witten invariants. This fact was announced in $|\overline{\mathrm{N} 08 \mathrm{c}}|$, and its proof is the subject of the present article.

\subsection{The lattice cohomology}

First we recall the definition of the lattice cohomology from [N08a] and [N10]. Let Char $:=\left\{k \in L^{\prime}:(k+l, l) \in 2 \mathbb{Z}\right.$ for all $\left.l \in L\right\}$ denote the set of characteristic elements of $L$. It is an $L^{\prime}$-torsor: Char $=K+2 L^{\prime}$.

The set of $q$-cubes, $\mathcal{Q}_{q}$, consists of pairs $(k, I) \in \operatorname{Char} \times \mathcal{P}(\mathcal{V}),|I|=q$ (here $\mathcal{P}(\mathcal{V})$ denotes the power set of $\mathcal{V}) . \square_{q}=(k, I)$ can be identified with the 'vertices' $\left(k+2 \sum_{j \in I^{\prime}} E_{j}\right)_{I^{\prime}}$, where $I^{\prime}$ runs over all subsets of $I$, of a $q$-cube in $L^{\prime} \otimes \mathbb{R}$. One defines the weight function induced by the intersection form

$$
w: \text { Char } \rightarrow \mathbb{Q}, \quad w(k):=-\left(k^{2}+|\mathcal{V}|\right) / 8,
$$

which extends to a weight function on the $q$-cubes,

$$
w\left(\square_{q}\right)=w((k, I))=\max _{I^{\prime} \subset I} w\left(k+2 \sum_{j \in I^{\prime}} E_{j}\right) .
$$


Let $\mathcal{F}_{q}$ be the direct product of $\mathbb{Z}_{\geq 0} \times \mathcal{Q}_{q}$ copies of $\mathbb{Z}$. We write the pair $(m, \square)$ as $U^{m} \square$. Then $\mathcal{F}_{q}$ becomes a $\mathbb{Z}[U]$-module by $U\left(U^{m} \square\right)=U^{m+1} \square$. One defines $\partial: \mathcal{F}_{q} \rightarrow \mathcal{F}_{q-1}$ as follows. For $\square=(k, I)=\left(k,\left\{v_{1}, \ldots, v_{q}\right\}\right)$ one sets

$\partial(k, I)=\sum_{l=1}^{q}(-1)^{l}\left(U^{w(k, I)-w\left(k, I \backslash v_{l}\right)}\left(k, I \backslash v_{l}\right)-U^{w(k, I)-w\left(k+2 E_{v_{l}}, I \backslash v_{l}\right)}\left(k+2 E_{v_{l}}, I \backslash v_{l}\right)\right)$.

Then $\partial \circ \partial=0$, hence $\left(\mathcal{F}_{*}, \partial\right)$ is a chain complex of $\mathbb{Z}[U]$-modules. The dual cochain complex is defined by $\mathcal{F}^{q}=\operatorname{Hom}_{\mathbb{Z}[U]}\left(\mathcal{F}_{q}, \mathcal{T}_{0}^{+}\right)$, consisting of finitely supported morphisms with $\phi\left(U^{m} \square\right)=U^{m} \phi(\square)$. Here, $\mathcal{T}_{0}^{+}$denotes the $\mathbb{Z}[U]$-module $\mathbb{Z}\left[U, U^{-1}\right] /$ $U \mathbb{Z}[U]$ with grading $\operatorname{deg}\left(U^{-d}\right)=2 d(d \geq 0)$, as usual. More generally, for any $r \in \mathbb{Q}$ one defines $\mathcal{T}_{r}^{+}$, the same module as $\mathcal{T}_{0}^{+}$, but graded (by $\mathbb{Q}$ ) in such a way that the $d+r$ homogeneous elements of $\mathcal{T}_{r}^{+}$are isomorphic to the $d$-homogeneous elements of $\mathcal{T}_{0}^{+}$.

$\mathcal{F}^{q}$ is a $\mathbb{Z}[U]$-module with a $\mathbb{Q}$-grading: $\phi \in \mathcal{F}^{q}$ is homogeneous of degree $r$ if for each $\square_{q} \in \mathcal{Q}_{q}$ with $\phi\left(\square_{q}\right) \neq 0, \phi\left(\square_{q}\right)$ is a homogeneous element of $\mathcal{T}_{0}^{+}$of degree $r-2 \cdot w\left(\square_{q}\right)$. The coboundary operator $\delta: \mathcal{F}^{q} \rightarrow \mathcal{F}^{q+1}$ is defined by $\delta(\phi)(\square)=$ $\phi(\partial(\square))$. The cohomology of $\left(\mathcal{F}^{*}, \delta\right)$ is the lattice cohomology of $\Gamma$, and it is denoted by $\mathbb{H}^{*}(\Gamma)$. Since the vertices of a cube belong to the same class Char $/ 2 L=K+2 L^{\prime} / 2 L$ (where a class has the form $[k]=\{k+2 l\}_{l \in L} \subset$ Char), the complex $\left(\mathcal{F}^{*}, \delta\right)$ and the cohomology $\mathbb{H}^{*}(\Gamma)$ have decompositions into natural direct sums of $\mathbb{Z}[U]$-modules:

$$
\left(\mathcal{F}^{*}, \delta\right)=\bigoplus_{[k] \in \operatorname{Char} / 2 L}\left(\mathcal{F}^{*}[k], \delta[k]\right) \quad \text { and } \quad \mathbb{H}^{*}(\Gamma)=\bigoplus_{[k] \in \operatorname{Char} / 2 L} \mathbb{H}^{*}(\Gamma,[k]) .
$$

In fact, if $\left[k_{1}\right]=\left[k_{2}\right]$ then $w\left(k_{1}\right)-w\left(k_{2}\right) \in \mathbb{Z}$, and the set of degrees of $\mathcal{F}^{*}[k]$ is $2 \mathbb{Z}$, shifted by a rational number. Since $\Gamma$ is negative definite, for each class $[k] \in$ Char $/ 2 L$ one has a well-defined rational number

$$
d[k]:=-\max _{k \in[k]} \frac{k^{2}+|\mathcal{V}|}{4}=2 \cdot \min _{k \in[k]} w(k) .
$$

One defines an augmentation $\epsilon: \mathcal{T}_{d[k]}^{+} \rightarrow \mathcal{F}^{0}[k]$ of the complex $\left(\mathcal{F}^{*}[k], \delta[k]\right)$; the cohomology of the augmented complex is called the reduced cohomology $\mathbb{H}_{\text {red }}^{*}(\Gamma,[k])$. One has $\mathbb{H}_{\text {red }}^{q}(\Gamma,[k]):=\mathbb{H}^{q}(\Gamma,[k])$ for $q>0$, and a decomposition into a direct sum of $\mathbb{Z}[U]$-modules:

$$
\mathbb{H}^{0}(\Gamma,[k])=\mathcal{T}_{d[k]}^{+} \oplus \mathbb{H}_{\text {red }}^{0}(\Gamma,[k]) .
$$

$\mathbb{H}_{\text {red }}^{*}(\Gamma,[k]):=\bigoplus_{q \geq 0} \mathbb{H}_{\text {red }}^{q}(\Gamma,[k])$ has finite $\mathbb{Z}$-rank. The 'normalized' Euler characteristic of $\mathbb{H}^{*}(\Gamma,[k])$ is

$$
e u\left(\mathbb{H}^{*}(\Gamma,[k])\right):=-d[k] / 2+\sum_{q}(-1)^{q} \operatorname{rank}_{\mathbb{Z}}\left(\mathbb{H}_{r e d}^{q}(\Gamma,[k])\right) .
$$

$\mathbb{H}^{*}(\Gamma)$ and $\mathbb{H}_{\text {red }}^{*}(\Gamma)$ depend only on $M=M(\Gamma)$, and not on the plumbing graph $\Gamma$. The involution $l^{\prime} \mapsto-l^{\prime}$ induces an isomorphism $\mathbb{H}^{*}(\Gamma,[k])=\mathbb{H}^{*}(\Gamma,[-k])$, hence

$$
e u\left(\mathbb{H}^{*}(\Gamma,[k])\right)=e u\left(\mathbb{H}^{*}(\Gamma,[-k])\right) .
$$


2.3.4. Rectangles. In combinatorial enumerations of the weighted $q$-cubes it is convenient to replace the set of all cubes by only those which are supported on a fixed compact subset of $L^{\prime} \otimes \mathbb{R}$. In the simplest case we take rectangles: for any fixed class $[k]=k_{0}+2 L \subset$ Char, one takes two characteristic elements $k_{1}, k_{2} \in[k]$ with $k_{1} \geq k_{2}$. We denote by $R=R\left(k_{1}, k_{2}\right)$ the rectangle $\left\{k \in[k]: k_{1} \geq k \geq k_{2}\right\}$. Similarly, for one fixed element $k_{1} \in[k]$, one can take $R=R\left(k_{1}\right)=\left\{k \in[k]: k_{1} \geq k\right\}$. Once such an $R$ is identified, one considers the complex $\left(\mathcal{F}^{*}(R), \delta(R)\right)$, constructed similarly to $\left(\mathcal{F}^{*}, \delta\right)$, consisting of all the cubes $(k, I)$ with all vertices in $R$ (this fact will be denoted by $(k, I) \in R)$. Using $\min (w \mid R):=\min _{k \in R} w(k)$ one also defines the corresponding augmented complex, and one gets the corresponding lattice cohomologies $\mathbb{H}^{*}(R,[k])$ and $\mathbb{H}_{\text {red }}^{*}(R,[k])$ with $\mathbb{H}^{0}(R,[k])=\mathcal{T}_{2 \min (w \mid R)}^{+} \oplus \mathbb{H}_{\text {red }}^{0}(R,[k])$. For more details, see [N08a]. We also define the 'normalized Euler characteristic' of this lattice cohomology, namely

$$
e u\left(\mathbb{H}^{*}(R,[k]):=-\min (w \mid R)+\sum_{q \geq 0}(-1)^{q} \operatorname{rank}_{\mathbb{Z}} \mathbb{H}_{r e d}^{q}(R,[k]) .\right.
$$

Let $\mathcal{S}_{s t}^{\prime}$ be the strict Lipman cone $\left\{l^{\prime} \in L^{\prime}:\left(l^{\prime}, E_{v}\right)<0\right.$ for all $\left.v\right\}$.

Proposition 2.3.5. Fix a class $[k]$. Assume that $k_{1} \in[k]$ satisfies $k_{1} \in-K+\mathcal{S}_{s t}^{\prime}$, that is, $\left(k_{1}, E_{v}\right) \leq e_{v}+1$ for any $v \in \mathcal{V}$. Then the following facts hold:

(a) For any $k \in[k], k>k_{1}$, there exists some $E_{v}$ in the support $\left|k-k_{1}\right|$ of $k-k_{1}$ such that $w\left(k-2 E_{v}\right) \leq w(k)$.

(b) There exists an increasing (computation) sequence $\left\{z_{n}\right\}_{n \geq 0}, z_{n} \in L$, with $z_{0}=0$, and $z_{n+1}=z_{n}+E_{v(n)}$ for some $v(n) \in \mathcal{V}$ when $n \geq 0$, satisfying:

(i) The coefficients of $z_{n}$ tend to infinity, that is, $-\lim _{n \rightarrow \infty}\left(z_{n}, E_{v}^{*}\right)=\infty$ for any $v$.

(ii) For fixed $n \geq 0$, let $x \in L$ be such that $x \leq z_{n}$ and $\left(x, E_{v(n)}^{*}\right)=\left(z_{n}, E_{v(n)}^{*}\right)$. Then $w\left(k_{1}+2 x\right) \leq w\left(k_{1}+2 x+2 E_{v(n)}\right)$.

(iii) The restriction $\mathbb{H}^{*}\left(R\left(k_{1}+2 z_{n+1}\right),[k]\right) \rightarrow \mathbb{H}^{*}\left(R\left(k_{1}+2 z_{n}\right)\right.$, [k]) is an isomorphism of weighted $\mathbb{Z}[U]$-modules compatible with the augmentation.

In particular, $\mathbb{H}^{*}(\Gamma,[k])=\mathbb{H}^{*}\left(R\left(k_{1}\right),[k]\right)$ compatibly with the augmentation.

Moreover, in a similar way, one can find $k_{2}$ (with all its $E_{v}$-coefficients sufficiently small) such that $\mathbb{H}^{*}(\Gamma,[k])=\mathbb{H}^{*}\left(R\left(k_{1}, k_{2}\right),[k]\right)$.

Proof. (a) Assume that $w\left(k-2 E_{v}\right)>w(k)$ for any $E_{v} \in\left|k-k_{1}\right|$. This says that $\left(k-2 E_{v}\right)^{2}<k^{2}$, that is, $\left(k, E_{v}\right)>E_{v}^{2}$, or $\left(k+K, E_{v}\right) \geq-1$. Since $\left(k_{1}+K, E_{v}\right) \leq-1$, one gets $\left(k-k_{1}, E_{v}\right) \geq 0$ for any $E_{v}$, hence $\left(k-k_{1}\right)^{2} \geq 0$, which is a contradiction.

(b) Fix a $D=\sum_{v} d_{v} E_{v} \in \mathcal{S}^{\prime} \cap L$ with $d_{v} \in \mathbb{Z}_{>0}$ for all $v$. By (a) we get an increasing computation sequence $\left\{y_{n}\right\}_{n=0}^{n_{0}}$ connecting 0 and $D$ so that $w\left(k_{1}+2 y_{n}\right) \leq w\left(k_{1}+2 y_{n+1}\right)$. Then the sequence $\left\{z_{n}\right\}_{n \geq 0}:=\left\{m D+y_{n}\right\}_{m \geq 0 ; 0 \leq n \leq n_{0}}$ satisfies (i) and $w\left(k_{1}+2 z_{n}\right) \leq$ $w\left(k_{1}+2 z_{n+1}\right)$. All other properties follow similarly to [N08a p. 518].

2.3.6. Counting weighted cubes and the Euler characteristic of the lattice cohomology. The next result generalizes the classical fact that the alternating sum of the number of $q$-cubes is the Euler characteristic of the cohomology. For any finite set $A \subset[k]$ define

$$
\mathcal{E}(A):=\sum_{(k, I) \in A}(-1)^{|I|+1} w((k, I)) \quad \text { and } \quad \mathcal{M}_{A}(t):=\sum_{(k, I) \in A}(-1)^{|I|} t^{w((k, I))} .
$$


Theorem 2.3.7. Let $R$ be a finite rectangle $R\left(k_{1}, k_{2}\right)$. Then $\mathcal{E}(R)=e u\left(\mathbb{H}^{*}(R,[k])\right)$.

Proof. We will reduce the result to the classical case via a certain geometric interpretation of the lattice cohomology from [N08a, (3.1.12)]. Note that $R$ can also be interpreted as a real rectangle in $L \otimes_{\mathbb{Z}} \mathbb{R}$ limited by the vertices $k_{1}$ and $k_{2}$. It has a natural cubic decomposition into the 'real cubes' $(k, I)$ with all vertices in the rectangle. For any nonnegative integer $n$ define $S_{n}$ as the union of all those real cubes of $L \otimes \mathbb{R}$ with vertices from $R$, and with weights $\leq n+\min (w \mid R)$. Let $\chi\left(S_{n}\right)$ be its (classical) Euler characteristic and $\chi_{\text {red }}\left(S_{n}\right)=\chi\left(S_{n}\right)-1$ the Euler characteristic of its reduced simplicial cohomology. Then, by [N08a, (3.1.12)], one has for any $q \geq 0$ the $\mathbb{Z}$-module isomorphisms

$$
\mathbb{H}^{q}(R,[k])=\bigoplus_{n \geq 0} H^{q}\left(S_{n}, \mathbb{Z}\right), \quad \mathbb{H}_{r e d}^{q}(R,[k])=\bigoplus_{n \geq 0} \widetilde{H}^{q}\left(S_{n}, \mathbb{Z}\right) .
$$

In particular, if we write $\mathcal{M}_{R}(t) /(1-t)$ as $\sum_{n \geq 0} a_{n} t^{n+\min (w \mid R)}$, then

$$
a_{n}=\sum_{\substack{(k, I) \in R \\ w((k, I)) \leq n+\min (w \mid R)}}(-1)^{|I|}=\chi\left(S_{n}\right) .
$$

Therefore,

$$
\frac{\mathcal{M}_{R}(t)-t^{\min (w \mid R)}}{1-t}=\sum_{n \geq 0} \chi_{\text {red }}\left(S_{n}\right) t^{n+\min (w \mid R)},
$$

hence, by 2.3.8,

$$
\lim _{t \rightarrow 1} \frac{\mathcal{M}_{R}(t)-t^{\min (w \mid R)}}{1-t}=\sum_{n \geq 0} \chi_{\text {red }}\left(S_{n}\right)=\sum_{q \geq 0}(-1)^{q} \operatorname{rank}_{\mathbb{Z}} \mathbb{H}_{r e d}^{q}(R,[k]),
$$

that is, $-\frac{d}{d t} \mathcal{M}_{R}(1)=e u\left(\mathbb{H}^{*}(R,[k])\right)$, the desired statement of the theorem.

2.3.9. Counting weighted cubes and the zeta function $Z(\mathbf{t})$. The next result provides the key step for the identification of the 'lattice cohomology package' and numerical invariants provided by the series $Z(\mathbf{t})$. Before we state it, let us recall that Char = $K+2 L^{\prime} \subset L^{\prime}$, hence $(k-K) / 2$ runs over $L^{\prime}$ when $k$ runs over Char.

Theorem 2.3.10. Let $\Gamma$ be a connected negative definite graph. Then

$$
Z(\mathbf{t})=\sum_{k \in \operatorname{Char}} \sum_{I \in \mathcal{P}(\mathcal{V})}(-1)^{|I|+1} w((k, I)) \mathbf{t}^{\frac{1}{2}(k-K)} .
$$

Since $\sum_{I}(-1)^{|I|}=0$, here $w((k, I))$ can be replaced by $w((k, I))+c$ for any constant $c$.

Proof. For each $k=\sum_{v} a_{v} E_{v}^{*} \in$ Char, where $a_{v} \equiv e_{v}(\bmod 2)$, write $\mathbf{z}^{a}:=\prod_{v} z_{v}^{a_{v}}$ and define the counting function

$$
\mathcal{U}_{\Gamma}(\mathbf{z}):=\sum_{k \in \operatorname{Char}} \sum_{I \in \mathcal{P}(\mathcal{V})}(-1)^{|I|+1} w((k, I)) \mathbf{z}^{a} .
$$


We determine $\mathcal{U}_{\Gamma}$ by induction on $|\mathcal{V}|$. If $|\mathcal{V}|=1$ and the decoration of the unique vertex is $e<0$, then $|e| E^{*}=E,\left(E^{*}\right)^{2}=1 / e$, and $k=a E^{*} \in$ Char with $a \equiv e(\bmod 2)$. Hence

$$
8 \mathcal{U}_{\Gamma}(z)=\sum_{a \equiv e(\bmod 2)}\left[-\frac{a^{2}}{|e|}+\max \left\{\frac{a^{2}}{|e|}, \frac{(a-2 e)^{2}}{|e|}\right\}\right] \cdot z^{a} .
$$

If $a \leq e$ then $a^{2} \geq(a-2 e)^{2}$, hence the coefficient vanishes. Otherwise we write $a=$ $e+2 n$ for $n \in \mathbb{Z}_{>0}$ and we get $\mathcal{U}(z)=\sum_{n \geq 1} n z^{e+2 n}=z^{e+2} /\left(1-z^{2}\right)^{2}$.

Next, we assume $|\mathcal{V}| \geq 2$. Let $u$ be a fixed end-vertex of $\Gamma$ (that is, $\delta_{u}=1$ ). Set $\Gamma_{0}:=\Gamma \backslash u$, the graph obtained by deleting $u$ and its supporting edge. If $k=\sum_{v} a_{v} E_{v}^{*} \in$ $\operatorname{Char}(\Gamma)$, we write $k_{0}=\sum_{v \neq u} a_{v} E_{v}^{*} \in \operatorname{Char}\left(\Gamma_{0}\right)$. The series $\mathcal{U}_{\Gamma}$ can be written as a sum $\mathcal{U}_{\Gamma}^{(1)}+\mathcal{U}_{\Gamma}^{(2)}$, where the first series is the sum over those subsets $I$ which do not contain $u$, while the second is the sum over the other terms. For the first case when $I \not \nexists u$,

$$
\mathcal{U}_{\Gamma}^{(1)}(\mathbf{z})=\mathcal{U}_{\Gamma_{0}}\left(\mathbf{z}_{0}\right) \cdot \sum_{a_{u} \equiv e_{u}(\bmod 2)} z_{u}^{a_{u}},
$$

where $\mathbf{z}_{0}$ are the variables $\left\{z_{v}\right\}_{v \neq u}$ corresponding to $\Gamma_{0}$. Indeed, one has $w((k, I))-$ $w(k)=w\left(\left(k_{0}, I\right)\right)-w\left(k_{0}\right)$ and $\sum_{I \not \supset u}(-1)^{|I|} w(k)=\sum_{I \not \supset u}(-1)^{|I|} w\left(k_{0}\right)=0$, hence

$$
\begin{aligned}
\sum_{k \in \operatorname{Char}(\Gamma)} \sum_{I \not \supset u} & (-1)^{|I|+1} w((k, I)) \mathbf{z}^{a} \\
& =\sum_{k \in \operatorname{Char}(\Gamma)} \sum_{I \not \supset u}(-1)^{|I|+1}(w((k, I))-w(k)) \mathbf{z}^{a} \\
& =\sum_{k \in \operatorname{Char}(\Gamma)} \sum_{I \not \supset u}(-1)^{|I|+1}\left(w\left(\left(k_{0}, I\right)\right)-w\left(k_{0}\right)\right) \mathbf{z}^{a} \\
& =\sum_{k_{0} \in \operatorname{Char}\left(\Gamma_{0}\right)} \sum_{a_{u} \equiv e_{u}(\bmod 2)} z_{u}^{a_{u}} \sum_{I \not \supset u}(-1)^{|I|+1} w\left(\left(k_{0}, I\right)\right) \prod_{v \neq u} z_{v}^{a_{v}} .
\end{aligned}
$$

In the second sum $u \in I$; set $I=I^{\prime} \cup u$ with $u \notin I^{\prime}$. Since

$$
\left(k+2 E_{I^{\prime}}+2 E_{u}\right)^{2}-\left(k+2 E_{I^{\prime}}\right)^{2}=4\left(-a_{u}+e_{u}+2\left(E_{I^{\prime}}, E_{u}\right)\right),
$$

where $\left(E_{I^{\prime}}, E_{u}\right) \in\{0,1\}$, one gets

$$
\left\{\begin{array}{l}
\text { if }-a_{u}+e_{u}<0, \text { then } w((k, I))=w\left(\left(k+2 E_{u}, I^{\prime}\right)\right) \\
\text { if }-a_{u}+e_{u} \geq 0, \text { then } w((k, I))=w\left(\left(k, I^{\prime}\right)\right)
\end{array}\right.
$$

Hence $\mathcal{U}_{\Gamma}^{(2)}(\mathbf{z})$ splits into two sums:

$$
\begin{aligned}
\sum_{k_{0} \in \operatorname{Char}\left(\Gamma_{0}\right)} \sum_{\substack{a_{u} \equiv e_{u}(\bmod 2) \\
a_{u} \leq e_{u}}} \sum_{I=I^{\prime} \cup u}(-1)^{|I|+1} w\left(\left(k, I^{\prime}\right)\right) \mathbf{z}^{a} \\
+\sum_{k_{0} \in \operatorname{Char}\left(\Gamma_{0}\right)} \sum_{a_{u} \equiv e_{u}(\bmod 2)} \sum_{I=I^{\prime} \cup u}(-1)^{|I|+1} w\left(\left(k+2 E_{u}, I^{\prime}\right)\right) \mathbf{z}^{a} .
\end{aligned}
$$


For the second one we use $E_{u}=-e_{u} E_{u}^{*}-E_{u_{0}}^{*}$, where $u_{0}$ is the adjacent vertex of $u$ in $\Gamma$. Then, computing both sums similarly to (2.3.14), we get

$$
\mathcal{U}_{\Gamma}^{(2)}(\mathbf{z})=-\mathcal{U}_{\Gamma_{0}}\left(\mathbf{z}_{0}\right) \cdot \sum_{\substack{a_{u} \equiv e_{u}(\bmod 2) \\ a_{u} \leq e_{u}}} z_{u}^{a_{u}}-\mathcal{U}_{\Gamma_{0}}\left(\mathbf{z}_{0}\right) \cdot z_{u_{0}}^{2} \cdot \sum_{\substack{a_{u} \equiv e_{u}(\bmod 2) \\ a_{u}>e_{u}}} z_{u}^{a_{u}}
$$

The contributions 2.3.13 and 2.3.15 combined provide

$$
\mathcal{U}_{\Gamma}(\mathbf{z})=\mathcal{U}_{\Gamma_{0}}\left(\mathbf{z}_{0}\right)\left(1-z_{u_{0}}^{2}\right) \cdot \sum_{\substack{a_{u} \equiv e_{u}(\bmod 2) \\ a_{u}>e_{u}}} z_{u}^{a_{u}}=\mathcal{U}_{\Gamma_{0}}\left(\mathbf{z}_{0}\right) \cdot \frac{1-z_{u_{0}}^{2}}{1-z_{u}^{2}} \cdot z_{u}^{e_{u}+2}
$$

This inductive step, together with the identity valid for $|\mathcal{V}|=1$, gives

$$
\mathcal{U}_{\Gamma}(\mathbf{z})=\prod_{v \in \mathcal{V}} z_{v}^{e_{v}+2} \cdot \prod_{v \in \mathcal{V}}\left(1-z_{v}^{2}\right)^{\delta_{v}-2} .
$$

From this and from the definition of $\mathcal{U}_{\Gamma}$ we obtain

$$
\sum_{k \in \operatorname{Char}} \sum_{I \in \mathcal{P}(\mathcal{V})}(-1)^{|I|+1} w((k, I)) \prod_{v \in \mathcal{V}} x_{v}^{\left(a_{v}-e_{v}-2\right) / 2}=\prod_{v \in \mathcal{V}}\left(1-x_{v}\right)^{\delta_{v}-2} .
$$

Then 2.3.11 follows via the substitution $x_{v}=\mathbf{t}^{E_{v}^{*}}$, since $K=\sum_{v}\left(2+e_{v}\right) E_{v}^{*}$.

\section{The proofs of Theorems A and B}

3.1. The definition of the invariant $\mathfrak{s}$ and its relation to $\mathbb{H}^{*}(\Gamma)$

Let $\Gamma$ be a graph as in 2.1 and $Z(\mathbf{t})$ the series defined in the introduction.

Theorem 3.1.1. (a) For any $l^{\prime} \in L^{\prime}$, the expression

$$
-\sum_{l \in L, l \nsupseteq 0} p_{l^{\prime}+l}-\frac{\left(K+2 l^{\prime}\right)^{2}+|\mathcal{V}|}{8}
$$

depends only on the class $\left[l^{\prime}\right] \in L^{\prime} / L$ of $l^{\prime}=\sum_{v \in \mathcal{V}} a_{v} E_{v}^{*}$, provided $a_{v} \geq-e_{v}-1$ for all $v \in \mathcal{V}$. (Since $Z$ is supported on $\mathcal{S}^{\prime}$, the sum in $\sqrt{3.1 .2}$ is finite by (2.1.2).)

(b) Consider the map $\mathfrak{s}: L^{\prime} / L \rightarrow \mathbb{Q},\left[l^{\prime}\right] \mapsto \mathfrak{s}_{\left[l^{\prime}\right]}$, where $\mathfrak{s}_{\left[-l^{\prime}\right]}$ is the expression 3.1.2). Then the set $\left\{\mathfrak{s}_{\left[l^{\prime}\right]}\right\}_{\left[l^{\prime}\right]}$ is independent of the negative definite plumbing representation $\Gamma$, it depends only on the oriented plumbed 3-manifold $M=M(\Gamma)$. In fact, for any $l^{\prime}$, one has

$$
\mathfrak{s}_{\left[-l^{\prime}\right]}=-e u\left(\mathbb{H}^{*}\left(\Gamma,\left[K+2 l^{\prime}\right]\right)\right) .
$$


Proof. We fix $k_{1}=K+2 l^{\prime}$ and $k_{2}$ as in Proposition 2.3.5, that is, $\left(k_{1}, E_{v}\right) \leq e_{v}+1$ and all the coefficients of $k_{2}$ are sufficiently small ( $k_{2}$ does not play an essential role, it only ensures the finiteness of the rectangles), and a computation sequence $\left\{z_{n}\right\}_{n \geq 0}$ as in Proposition 2.3.5. b). Set

$$
R^{\prime}:=\left\{k \in[k]: k \geq k_{2}, k-k_{1}=\sum l_{v} E_{v} \text { so that } \exists l_{v} \leq 0\right\} .
$$

Although $R^{\prime}$ is not finite, $R^{\prime} \cap \mathcal{S}^{\prime}$ is finite by 2.1.2). Fix some $\tilde{n}$ so that $R^{\prime} \cap \mathcal{S}^{\prime} \subset$ $R\left(k_{1}+2 z_{\tilde{n}}, k_{2}\right)$ and define $\widetilde{R}:=R^{\prime} \cap R\left(k_{1}+2 z_{\tilde{n}}, k_{2}\right)$. Take also

$$
\text { ว } \widetilde{R}:=\left\{k \in[k]: k_{1} \leq k \leq k_{1}+2 z_{\tilde{n}}, k-k_{1}=\sum l_{v} E_{v} \text { so that } \exists l_{v}=0\right\} .
$$

Then, by Theorem 2.3 .10 .

$$
\sum_{l \in L, l \ngtr 0} p_{l^{\prime}+l}=\mathcal{E}(\widetilde{R})-\mathcal{E}(\partial \widetilde{R}) .
$$

Now, we claim that by combinatorial cancellation in the sum, $\mathcal{E}(\widetilde{R})=\mathcal{E}(R)$, where $R=R\left(k_{1}, k_{2}\right)$. This follows by induction using the sequence $\left\{z_{n}\right\}_{0 \leq n \leq n}$, since $\mathcal{E}\left(R^{\prime} \cap R\left(k_{1}+2 z_{n+1}, k_{2}\right)\right)=\mathcal{E}\left(R^{\prime} \cap R\left(k_{1}+2 z_{n}, k_{2}\right)\right)$. Indeed, for any $I$ containing $v(n)$ and cube $(k, I) \in R^{\prime} \cap\left(R\left(k_{1}+2 z_{n+1}, k_{2}\right) \backslash R\left(k_{1}+2 z_{n}, k_{2}\right)\right)$ one has $w((k, I))=w\left(\left(k+2 E_{v(n)}, I \backslash v(n)\right)\right)$ by Proposition 2.3.5 (b)(ii). Similarly, one gets $\mathcal{E}(\partial \widetilde{R})=-w\left(k_{1}\right)$. Hence 3.1 .4 reads

$$
-w\left(K+2 l^{\prime}\right)+\sum_{l \in L, l \nsupseteq 0} p_{l^{\prime}+l}=\mathcal{E}(R) .
$$

The right hand side is $\operatorname{eu}\left(\mathbb{H}^{*}\left(R,\left[k_{1}\right]\right)\right)$ by Theorem 2.3.7, which equals $e u\left(\mathbb{H}^{*}\left(\Gamma,\left[k_{1}\right]\right)\right)$ by Proposition 2.3.5 In particular, the left hand side too depends only on $\left[l^{\prime}\right]$. It is invariant under blow up of the graph since so is the lattice cohomology (cf. [N08a, ,N10]).

\subsection{The surgery formula for $\mathfrak{s}$}

3.2.1. The additivity formula. Similarly to the proof of Theorem 2.3 .10 , we change the variables of the series $Z(\mathbf{t})$. By setting $x_{v}:=\mathbf{t}^{E_{v}^{*}}$ for all $v \in \mathcal{V}, Z(\mathbf{t})$ transforms into $\mathcal{Z}_{\Gamma}(\mathbf{x})=\prod_{v \in \mathcal{V}}\left(1-x_{v}\right)^{\delta_{v}-2}$, whose Taylor series at the origin is denoted by $\sum q_{a} \mathbf{x}^{a}$, where $\mathbf{x}^{a}=x_{1}^{a_{1}} \cdots x_{s}^{a_{s}}$. The exponents $a_{v}$ are the coordinates of $L^{\prime}$ in the basis $\left\{E_{v}^{*}\right\}_{v}$, i.e. if $l^{\prime}=\sum_{v} a_{v} E_{v}^{*}$ then $a_{v}=-\left(l^{\prime}, E_{v}\right)$ and $\mathbf{t}^{l^{\prime}}$ transforms into $\mathbf{x}^{a}$. In particular, $q_{a}=p_{l^{\prime}}$, and we also use the notation $a$ for $l^{\prime} \in L^{\prime}$. For any fixed $l^{\prime}=\sum_{v} a_{v} E_{v}^{*}$ we define

$$
h_{a}^{\Gamma}:=\sum_{l \in L, l \nsupseteq 0} p_{l^{\prime}+l}=\sum_{b \in S_{\Gamma}(a)} q_{b}^{\Gamma},
$$

where $S_{\Gamma}(a)=\left\{b \in L^{\prime}(\Gamma): b=a+\sum n_{v} E_{v}, n_{v} \in \mathbb{Z}, \exists n_{v}<0\right\}$. We assume that $s \geq 2$ and we fix an end-vertex $u$ of $\Gamma$. We set

$$
h_{a}^{u}:=\sum q_{b}^{\Gamma} \quad\left(\text { sum over } b=a+\sum n_{v} E_{v}, n_{v} \in \mathbb{Z}, n_{u}<0\right) .
$$


The inclusion of the subgraph $\Gamma \backslash u$ induces $i: L(\Gamma \backslash u) \rightarrow L(\Gamma), i\left(E_{v}\right) \mapsto E_{v}$ (the symbol $i$ is sometimes omitted), and its dual, the restriction $R: L^{\prime}(\Gamma) \rightarrow L^{\prime}(\Gamma \backslash u)$ with

$$
\left\{\begin{array}{l}
R\left(E_{v}^{*, \Gamma}\right)=E_{v}^{*, \Gamma \backslash u} \quad \text { for } v \neq u, \quad \text { and } \quad R\left(E_{u}^{*, \Gamma}\right)=0 \\
R\left(E_{v}^{\Gamma}\right)=E_{v}^{\Gamma \backslash u} \quad \text { for } v \neq u, \quad \text { and } \quad R\left(E_{u}^{\Gamma}\right)=-E_{w}^{*, \Gamma \backslash u}
\end{array}\right.
$$

where $w$ is the adjacent vertex of $u$. We abbreviate $R\left(\sum_{v} a_{v} E_{v}^{*}\right)$ as $R(a)$.

Proposition 3.2.4. Assume that $w$, the adjacent vertex of $u$ in $\Gamma$, has valency two. Then, if $a_{u} \gg 0$ (compared with $\left.R(a)\right)$, one has

$$
h_{a}^{\Gamma}=h_{a}^{u}+h_{R(a)}^{\Gamma \backslash u} .
$$

Proof. Since $\delta_{w}=2, \mathcal{Z}_{\Gamma}$ and $\mathcal{Z}_{\Gamma \backslash u}$ have the form

$$
\mathcal{Z}_{\Gamma}=\tilde{\mathcal{Z}} \cdot \frac{1}{1-x_{u}}, \quad \mathcal{Z}_{\Gamma \backslash u}=\tilde{\mathcal{Z}} \cdot \frac{1}{1-x_{w}},
$$

where $\widetilde{\mathcal{Z}}$ does not depend on the variables $x_{w}$ and $x_{u}$. Therefore, for any relevant $b \in$ $S_{\Gamma}(a)$ (i.e. when $q_{b}^{\Gamma} \neq 0$ ) one has $b_{w}=0$ and $b_{u} \geq 0$. Hence

$$
h_{a}^{\Gamma}-h_{a}^{u}=\sum_{b \in S_{\Gamma}^{\prime}(a)} q_{b}^{\Gamma}
$$

where $S_{\Gamma}^{\prime}(a)=\left\{b \in S_{\Gamma}(a): b_{w}=0, b_{u} \geq 0, n_{u} \geq 0, \exists n_{v}<0\right\}$.

By a similar argument based on $(3.2 .5)$, for any relevant $c \in S_{\Gamma \backslash u}(R(a))$, one has $c_{w} \geq 0$, hence it is enough to consider the subset $S_{\Gamma \backslash u}^{\prime}(R(a))=\left\{c \in S_{\Gamma \backslash u}(R(a))\right.$ : $\left.c_{w} \geq 0\right\}$ in the computation of $h_{R(a)}^{\Gamma \backslash u}$. Applying $\left(\cdot, E_{u}\right)$ to the identity $b=a+\sum_{v} n_{v} E_{v}$, one gets

$$
b_{u}=a_{u}-n_{w}-I_{u u} n_{u} .
$$

Since $n_{u}=-\left(E_{u}^{*, \Gamma}, b-a\right)$ in terms of $b$, for any fixed $a$ one has a well-defined map

$$
\Phi: S_{\Gamma}^{\prime}(a) \rightarrow S_{\Gamma \backslash u}^{\prime}(R(a)), \quad b=\left(\left\{b_{v}\right\}_{v \neq w, u}, b_{w}=0, b_{u}\right) \mapsto\left(\left\{b_{v}\right\}_{v \neq w, u}, n_{u}\right) .
$$

Here, $n_{u}$ maps to the $w$-entry in $S_{\Gamma \backslash u}^{\prime}(R(a))$. In other words, $\Phi(b)=R(b)+$ $n_{u} E_{w}^{*, \Gamma \backslash u}$ (use 3.2 .3 ). Moreover, again by $3.2 .3, \Phi(b)-R(a)=R\left(\sum_{v \neq u} n_{v} E_{v}^{\Gamma}\right)=$ $\sum_{v \neq u} n_{v} E_{v}^{\Gamma \backslash u}$, hence the integers $\left\{n_{v}\right\}_{v \neq u}$ are the same at the level of $\Gamma$ and $\Gamma \backslash u$. This fact and 3.2.6) imply the injectivity of $\Phi$. For the surjectivity, for any $\left(\left\{b_{v}\right\}_{v \neq w, u}, n_{u}\right) \in$ $S_{\Gamma \backslash u}^{\prime}(R(a))$ define $b_{u}$ by 3.2 .6$)$. Then $\left(\left\{b_{v}\right\}_{v \neq w, u}, 0, b_{u}\right)$ automatically satisfies all the conditions of $S_{\Gamma}^{\prime}(a)$, except maybe one, namely $b_{u} \geq 0$. In order to guarantee this one too, we argue as follows: for $R(a)$ fixed, consider all the elements $\left(\left\{b_{v}\right\}_{v \neq w, u}, n_{u}\right) \in$ $S_{\Gamma \backslash u}^{\prime}(R(a))$, and associate with them the maximum $M$ of all the possible values $n_{w}+I_{u u} n_{u}$. Then, if we take $a_{u} \geq M$, then by 3.2.6 the inequality $b_{u} \geq 0$ is also satisfied. 
Finally, notice that from 3.2 .5 , for any $b \in S_{\Gamma}^{\prime}(a)$ one has

$$
q_{b}^{\Gamma}=q_{\Phi(b)}^{\Gamma \backslash u}
$$

since both coincide with the $\prod_{v \neq w, u} x^{b_{v}}$-coefficient of $\widetilde{\mathcal{Z}}$.

Corollary 3.2.7. Fix an end-vertex $u$ as above. For $l^{\prime}=\sum_{v} a_{v} E_{v}^{*, \Gamma}$ with all $a_{v}$ large (and $a_{u}$ large compared with the others), one has

$$
h_{a}^{u}=-\mathfrak{s}_{\left[-l^{\prime}\right]}^{\Gamma}-\frac{K_{\Gamma}^{2}+|\mathcal{V}|}{8}+\mathfrak{s}_{\left[-R\left(l^{\prime}\right)\right]}^{\Gamma \backslash u}+\frac{K_{\Gamma \backslash u}^{2}+|\mathcal{V} \backslash u|}{8}-\frac{\left(l^{\prime}, E_{u}^{*}\right) \cdot\left(l^{\prime}+K_{\Gamma}, E_{u}^{*}\right)}{2\left(E_{u}^{*}, E_{u}^{*}\right)} .
$$

Proof. Theorem 3.1.1 applied for $l^{\prime}$ and $R\left(l^{\prime}\right)$, and Proposition 3.2.4 provide

$$
h_{a}^{u}=-\mathfrak{s}_{\left[-l^{\prime}\right]}^{\Gamma}-\frac{\left(K_{\Gamma}+2 l^{\prime}\right)^{2}+|\mathcal{V}|}{8}+\mathfrak{s}_{\left[-R\left(l^{\prime}\right)\right]}^{\Gamma u}+\frac{\left(K_{\Gamma \backslash u}+2 R\left(l^{\prime}\right)\right)^{2}+|\mathcal{V} \backslash u|}{8} .
$$

Then use the identity $K_{\Gamma \backslash u}=R\left(K_{\Gamma}\right)$, and

$$
l^{\prime \prime}=i R\left(l^{\prime \prime}\right)+\frac{\left(l^{\prime \prime}, E_{u}^{*}\right)}{\left(E_{u}^{*}, E_{u}^{*}\right)} \cdot E_{u}^{*}
$$

for both $l^{\prime \prime}=l^{\prime}$ and $l^{\prime \prime}=K_{\Gamma}$, and $\left(i(l), E_{u}^{*}\right)=0$ for any $l \in L^{\prime}(\Gamma \backslash u)$.

3.2.8. The series $\mathcal{H}_{\left[l^{\prime}\right], u}(t)$ and its periodic constant. For any series $S(\mathbf{t}) \in \mathbb{Z}\left[\left[L^{\prime}\right]\right]$, $S(\mathbf{t})=\sum_{l^{\prime}} c_{l^{\prime}} \mathbf{t}^{l^{\prime}}$, we have the natural decomposition

$$
S=\sum_{h \in L^{\prime} / L} S_{h}, \quad \text { where } \quad S_{h}:=\sum_{l^{\prime}:\left[l^{\prime}\right]=h} c_{l^{\prime}} \mathbf{t}^{l^{\prime}} .
$$

In particular, for any fixed class $\left[l^{\prime}\right] \in L^{\prime} / L$, one can consider the component $Z_{\left[l^{\prime}\right]}(\mathbf{t})$ of $Z(\mathbf{t})$. In fact (see e.g. [N08b, (3.1.20)]),

$$
Z_{\left[l^{\prime}\right]}(\mathbf{t})=\frac{1}{d} \sum_{\rho \in\left(L^{\prime} / L\right)^{-}} \rho\left(\left[l^{\prime}\right]\right)^{-1} \cdot \prod_{v \in \mathcal{V}}\left(1-\rho\left(\left[E_{v}^{*}\right]\right) \mathbf{t}^{E_{v}^{*}}\right)^{\delta_{v}-2},
$$

where $\left(L^{\prime} / L\right)^{-}$is the Pontryagin dual of $L^{\prime} / L$, and $d=\operatorname{det}(-I)=\left|L^{\prime} / L\right|$.

Definition 3.2.10. For any class $\left[l^{\prime}\right] \in L^{\prime} / L$ and vertex $u \in \mathcal{V}$ of $\Gamma$ set

$$
\mathcal{H}_{\left[l^{\prime}\right], u}(t):=\left.Z_{\left[l^{\prime}\right]}(\mathbf{t})\right|_{\substack{t_{u}=t^{d} \\ t_{v}=1 \text { for } v \neq u}} \in \mathbb{Z}[[t]] .
$$

Definition 3.2.11 ([NO09, 3.9], [008, 4.8(1)]). Let $S(t)=\sum_{i \geq 0} c_{i} t^{i}$ be a formal power series. Suppose that for some positive integer $p$, the expression $\sum_{i=0}^{p n-1} c_{i}$ is a polynomial $P_{p}(n)$ in the variable $n$. Then the constant term of $P_{p}(n)$ is independent of $p$. We call this constant term the periodic constant of $S$ and denote it by $\operatorname{pc}(S)$. 
3.2.12. The surgery formula for $\mathfrak{s}$. The relation between the coefficients $h_{a}^{u}$ defined in 3.2.1 and the series $\mathcal{H}_{\left[l^{\prime}\right], u}(t)$ is as follows (below, $\{r\}$ will denote the fractional part of the rational number $r$ ):

Theorem 3.2.13. Consider the graph $\Gamma$ and let $u$ be one of its end-vertices. Fix $l^{\prime}=$ $\sum_{v} a_{v} E_{v}^{*}=\sum_{v} l_{v}^{\prime} E_{v}$ with a as in 3.2.7 Abbreviate $l_{u}^{\prime}$ to $\ell$. Then:

(a) If $\mathcal{H}_{\left[l^{\prime}\right], u}(t)=\sum_{i \geq 0} c_{i} t^{i}$ then $h_{a}^{u}=\sum_{i<d \ell} c_{i}$.

(b) Take $\bar{l}^{\prime} \in L^{\prime}$ such that $\left(\bar{l}^{\prime}, E_{u}^{*}\right) \in(-1,0]$. Then

$$
\operatorname{pc}\left(\mathcal{H}_{\left[\bar{l}^{\prime}\right], u}\right)=-\mathfrak{s}_{\left[-\bar{l}^{\prime}\right]}^{\Gamma}-\frac{\left(K_{\Gamma}+2{\overline{l^{\prime}}}^{2}+|\mathcal{V}|\right.}{8}+\mathfrak{s}_{[-R \backslash u}^{\left.\Gamma\left(\bar{l}^{\prime}\right)\right]}+\frac{\left(K_{\Gamma \backslash u}+2 R\left(\bar{l}^{\prime}\right)\right)^{2}+|\mathcal{V} \backslash u|}{8} .
$$

Proof. Write $Z_{\left[l^{\prime}\right]}(\mathbf{t})=\sum c_{l^{\prime \prime}} \mathbf{t}^{l^{\prime \prime}}$. Then (a) follows from $\mathcal{H}_{\left[l^{\prime}\right], u}(t)=\sum c_{l^{\prime \prime}} d l^{\prime \prime}$ and

$$
h_{a}^{u}=\sum_{b: n_{u}<0} q_{b}^{\Gamma}=\sum_{l^{\prime \prime}: l_{u}^{\prime \prime}<l_{u}^{\prime}} c_{l^{\prime \prime}}
$$

(b) For any fixed $\bar{l}^{\prime}$ as in the assumption of (b), take $l^{\prime}$ such that $\left[l^{\prime}\right]=\left[\bar{l}^{\prime}\right]$, and $\left[R\left(l^{\prime}\right)\right]=\left[R\left(\overline{l^{\prime}}\right)\right]$ and $l^{\prime}=\sum_{v} a_{v} E_{v}^{*}=\sum_{v} l_{v}^{\prime} E_{v}$ with $a \gg 0$. Since $\bar{l}^{\prime}-l^{\prime} \in L$, we find that $l_{u}^{\prime}+\left(\bar{l}^{\prime}, E_{u}^{*}\right)=\left(\bar{l}^{\prime}-l^{\prime}, E_{u}^{*}\right) \in \mathbb{Z}$, hence $-\left(\bar{l}^{\prime}, E_{u}^{*}\right)$ is the fractional part of $l_{u}^{\prime}$. Write $l_{u}^{\prime}=\ell$ and let $n$ be its integral part. For this $l^{\prime}$ we apply part (a) and Corollary 3.2.7

In order to compute the periodic constant of $\mathcal{H}_{\left[l^{\prime}\right], u}(t)$, notice that if $c_{l^{\prime \prime}} \neq 0$ then $l^{\prime \prime}-l^{\prime} \in L$, hence $l_{u}^{\prime \prime}-l_{u}^{\prime}=\left(E_{u}^{*}, l^{\prime}-l^{\prime \prime}\right) \in \mathbb{Z}$. Therefore, if the coefficient $c_{i}$ of $\mathcal{H}_{\left[l^{\prime}\right], u}$ is nonzero, then $i-d\{\ell\} \in d \mathbb{Z}$. In particular, for $c_{i} \neq 0, i<d \ell$ if and only if $i \leq d n-1$. This shows that one has to write $h_{a}^{u}$ as $P(n)$ for some polynomial $P$, and then the periodic constant of $\mathcal{H}_{\left[l^{\prime}\right], u}$ is $P(0)$. We deduce that $P(n)$ is the right hand side of the identity of Corollary 3.2.7 after the substitution $\left(l^{\prime}, E_{u}^{*}\right)=-l_{u}^{\prime}=-\{\ell\}-n$. Therefore

$\operatorname{pc}\left(\mathcal{H}_{\left[l^{\prime}\right], u}\right)=-\mathfrak{s}_{\left[-l^{\prime}\right]}^{\Gamma}-\frac{K_{\Gamma}^{2}+|\mathcal{V}|}{8}+\mathfrak{s}_{\left[-R\left(l^{\prime}\right)\right]}^{\Gamma \backslash u}+\frac{K_{\Gamma \backslash u}^{2}+|\mathcal{V} \backslash u|}{8}-\frac{\{\ell\} \cdot\left(\{\ell\}-\left(K_{\Gamma}, E_{u}^{*}\right)\right)}{2\left(E_{u}^{*}, E_{u}^{*}\right)}$.

Now, this identity provides (b) by a straightforward computation (similar to the computation from the proof of 3.2.7.

Strictly speaking, the above argument proves the surgery formula (b) only if $\delta_{w}=2$ (cf. the assumption of Proposition 3.2.4). In general we argue as follows: let $u$ be an endvertex, and let us blow up the unique edge adjacent to $u$ getting in this way $\Gamma^{b}$. Then the newly created vertex $w$ has $\delta_{w}=2$. Hence in this situation we can apply the above proof for $\Gamma^{b}$ and $\Gamma^{b} \backslash u$. Since blowing down the $w$-vertex in $\Gamma^{b} \backslash u$ we get $\Gamma \backslash u$, and all the invariants involved in (b) are stable with respect to blow up/down, the result follows. Indeed,

$$
\left(K+2 l^{\prime}\right)^{2}+|\Gamma|=\left(K^{b}+2 \pi^{*}\left(l^{\prime}\right)\right)^{2}+\left|\Gamma^{b}\right|,
$$

the $\mathfrak{s - t e r m s}$ are stable by Theorem $3.1 .1(\mathrm{~b})$, and $\mathcal{H}_{\left[l^{\prime}\right], u}$ by the fact that it depends only on the numbers $\left(E_{v_{1}}^{*}, E_{v_{2}}^{*}\right)$ where $\delta_{v_{i}} \neq 2$. 


\subsection{The identification of $\mathfrak{s}$ with the Seiberg-Witten invariant}

3.3.1. Some facts about the Seiberg-Witten invariant of $M$. Let $\Gamma$ be a connected negative definite plumbing graph, and let $\widetilde{X}$ be the plumbed 4-manifold constructed from $\Gamma$. If $\Gamma$ is a resolution graph, e.g. as in Subsection 2.1 then the (diffeomorphism type of the) resolution serves for $\widetilde{X}$. Let $\tilde{\sigma}_{\text {can }}$ be the canonical $\operatorname{spin}^{c}$-structure on $\widetilde{X}$; its first Chern class $c_{1}\left(\widetilde{\sigma}_{c a n}\right)$ is $-K \in L^{\prime}$ (cf. [GS99, p. 415]). The set of $\operatorname{spin}^{c}$-structures $\operatorname{Spin}^{c}(\widetilde{X})$ is an $L^{\prime}$-torsor; if we denote the $L^{\prime}$-action by $l^{\prime} * \widetilde{\sigma}$, then $c_{1}\left(l^{\prime} * \widetilde{\sigma}\right)=c_{1}(\widetilde{\sigma})+2 l^{\prime}$.

If $M=M(\Gamma)$ is the plumbed 3-manifold associated with $\Gamma$, then $M=\partial \widetilde{X}$, and all the $\operatorname{spin}^{c}$-structures of $M$ are obtained by restrictions from $\widetilde{X} . \operatorname{Spin}^{c}(M)$ is an $L^{\prime} / L$ torsor, compatible with the restriction and the projection $L^{\prime} \rightarrow L^{\prime} / L$. The canonical $\operatorname{spin}^{c}$-structure $\sigma_{\text {can }}$ of $M$ is the restriction of $\widetilde{\sigma}_{\text {can }}$.

We denote the Seiberg-Witten invariant by $\mathfrak{s w}: \operatorname{Spin}^{c}(M) \rightarrow \mathbb{Q}, \sigma \mapsto \mathfrak{s w}_{\sigma}$. Next we recall a surgery formula satisfied by them, proved in [BN10].

Let us fix one of the end-vertices $u$ of $\Gamma$ (though the statement is true for any vertex, cf. loc.cit). Let $\widetilde{X}(\Gamma \backslash u)$ be the tubular neighbourhood of $\bigcup_{v \neq u} E_{v}$ in $\widetilde{X}$, and $M_{u}$ its boundary. Hence, $M_{u}$ is the plumbed 3-manifold associated with $\Gamma \backslash u$.

Fix any $\sigma \in \operatorname{Spin}^{c}(M)$, extend it to a $\operatorname{spin}^{c}$-structure $\widetilde{\sigma} \in \operatorname{Spin}^{c}(\tilde{X})$ of the form $\widetilde{\sigma}=\widetilde{l}^{\prime} * \widetilde{\sigma}_{c a n}$ with $\widetilde{l}^{\prime} \in L^{\prime},\left(\widetilde{l}^{\prime}, E_{u}^{*}\right) \in[0,1)$. Then consider $R\left(\widetilde{l^{\prime}}\right) \in L^{\prime}(\Gamma \backslash u)$ and the restrictions $\widetilde{\sigma}_{u}$ and $\sigma_{u}$ of $\tilde{\sigma}$ to $\tilde{X}(\Gamma \backslash u)$ and $\partial \tilde{X}(\Gamma \backslash u)$ respectively. Then the main result of [BN10] is the following identity. (Here we wish to draw the reader's attention to the notational differences between the present note and [BN10]: in that article the 'dual' $E_{v}^{*}$ has opposite sign, this creates a sign difference in $\left.\widetilde{l^{\prime}}, E_{u}^{*}\right) \in[0,1)$, and also in the expression of $\mathcal{H}$ from 3.2 .9 the characters $\rho \in\left(L^{\prime} / L\right)^{\top}$ should be replaced by their inverses. Hence, $\mathcal{H}_{\sigma, u}$ of [BN10] is our $\mathcal{H}_{\left[-\widetilde{l}^{\prime}\right], u}$.

Theorem 3.3.2 ([BN10] $)$.

$$
\mathfrak{s w}_{\sigma}(M)+\frac{c_{1}(\widetilde{\sigma})^{2}+|\mathcal{V}|}{8}=-\operatorname{pc}\left(\mathcal{H}_{\left[-\widetilde{l}^{\prime}\right], u}\right)+\mathfrak{s w}_{\sigma_{u}}\left(M_{u}\right)+\frac{c_{1}\left(\widetilde{\sigma}_{u}\right)^{2}+|\mathcal{V} \backslash u|}{8}
$$

3.3.3. Proof of Theorem A. This is completed by the next result.

Theorem 3.3.4. Let $\mathfrak{s}: L^{\prime} / L \rightarrow \mathbb{Q}$ be the invariant defined from the series $Z(\mathbf{t})$ in 3.1 .2 Then for any $\left[l^{\prime}\right] \in L^{\prime} / L$ one has

$$
\mathfrak{s}_{\left[l^{\prime}\right]}=\mathfrak{s w}_{\left[l^{\prime}\right] * \sigma_{\text {can }}} .
$$

Proof. Notice that $-\bar{l}^{\prime}$ considered in Theorem 3.2 .13 satisfies the needed assumptions for $\widetilde{l}^{\prime}$ in 3.3.1. Moreover, $c_{1}(\widetilde{\sigma})=-\left(2 \bar{l}^{\prime}+K\right)$, and $c_{1}\left(\widetilde{\sigma}_{u}\right)=-R\left(2 \bar{l}^{\prime}+K\right)$, and $\sigma=$ $\left[-\bar{l}^{\prime}\right] * \sigma_{\text {can }}$. Hence, the surgery identities in Theorems 3.3.2 and 3.2.13 (b) show that $\mathfrak{s}_{\left[-l^{\prime}\right]}$ satisfies the same surgery formula as $\mathfrak{s w}_{\left[-l^{\prime}\right] * \sigma_{c a n}}$. One can verify that they coincide for graphs with one vertex (or one can apply [N08c, §4] which shows that they coincide for links of splice-quotient singularities, including all Seifert manifolds). Therefore, by induction on the number of vertices, we get the result.

3.3.5. Proof of Theorem B. Combine 2.3.3, 3.1.3 and Theorem 3.3.4. 


\section{References}

[BN10] Braun, G., Némethi, A.: Surgery formula for Seiberg-Witten invariants of negative definite plumbed 3-manifolds. J. Reine Angew. Math. 638, 189-208 (2010) Zbl pre05667750 MR 2595340

[CDG04] Campillo, A., Delgado, F., Gusein-Zade, S. M.: Poincaré series of a rational surface singularity. Invent. Math. 155, 41-53 (2004) Zbl 1060.14054 MR 2025300

[CDG08] Guse ̌n-Zade, S. M., Delgado, F., Campillo, A.: Universal abelian covers of rational surface singularities, and multi-index filtrations. Funktsional. Anal. i Prilozhen. 42, no. 2, 3-10 (2008) (in Russian) Zbl 1156.14317 MR 2438013

[CHR04] Cutkosky, S. D., Herzog, J., Reguera, A.: Poincaré series of resolutions of surface singularities. Trans. Amer. Math. Soc. 356, 1833-1874 (2004) Zbl 1076.14006 MR 2031043

[GS99] Gompf, R. E., Stipsicz, A. I.: 4-Manifolds and Kirby Calculus. Grad. Stud. Math. 20, Amer. Math. Soc. (1999) Zbl 0933.57020 MR 1707327

[L96] Lescop, C.: Global Surgery Formula for the Casson-Walker Invariant. Ann. of Math. Stud. 140, Princeton Univ. Press (1996) Zbl 0949.57008 MR 1372947

[MW02] Marcolli, M., Wang, B. L.: Seiberg-Witten invariant and the Casson-Walker invariant for rational homology 3-spheres. In: Proc. Euroconf. on Partial Differential Equations and their Applications to Geometry and Physics (Castelvecchio Pascoli, 2000), Geom. Dedicata 91, 45-58 (2002) Zbl 0994.57027 MR 1919892

[N03] Némethi, A.: Line bundles associated with normal surface singularities. arXiv:math/0310084; published as part of [N07]

[N05] Némethi, A.: On the Ozsváth-Szabó invariant of negative definite plumbed 3-manifolds. Geom. Topol. 9, 991-1042 (2005) Zbl 1138.57301 MR 2140997

[N07] Némethi, A.: Graded roots and singularities. In: Singularities in Geometry and Topology, World Sci., Hackensack, NJ, 394-463 (2007) Zbl 1125.14019 MR 2311495

[N08a] Némethi, A.: Lattice cohomology of normal surface singulariites. Publ. RIMS Kyoto Univ. 44, 507-543 (2008) Zbl 1149.14029 MR 2426357

[N08b] Némethi, A.: Poincaré series associated with surface singularities. In: Singularities I: Algebraic and Analytic Aspects, Contemp. Math. 474, Amer. Math. Soc., 271-299 (2008) Zbl 1162.32004 MR 2454352

[N08c] Némethi, A.: The cohomology of line bundles of splice-quotient singularities. arXiv:0810.4129

[N10] Némethi, A.: Two exact sequences for lattice cohomology. In: Proceedings dedicated to H. Moscovici's 65th birthday, to appear; arXiv:1001.0640

[NN02] Némethi, A., Nicolaescu, L. I.: Seiberg-Witten invariants and surface singularities. Geom. Topol. 6, 269-328 (2002) Zbl 1031.32023 MR 1914570

[NO09] Némethi, A., Okuma, T.: On the Casson invariant conjecture of Neumann-Wahl. J. Algebraic Geom. 18, 135-149 (2009) Zbl 1154.14025 MR 2448281

[Ni04] Nicolaescu, L. I.: Seiberg-Witten invariants of rational homology 3-spheres. Comm. Contemp. Math. 6, 833-866 (2004) Zbl 1082.57010 MR 2111431

[O08] Okuma, T.: The geometric genus of splice quotient singularities. Trans. Amer. Math. Soc. 360, 6643-6659 (2008) Zbl 1162.32017 MR 2434304

[OSz03a] Ozsváth, P. S., Szabó, Z.: Absolutely graded Floer homologies and intersection forms for four-manifolds with boundaries. Adv. Math. 173, 179-261 (2003) Zbl 1025.57016 MR 1957829

[OSz03b] Ozsváth, P. S., Szabó, Z.: On the Floer homology of plumbed three-manifolds. Geom. Topol. 7, 185-224 (2003) Zbl 1130.57302 MR 1988284

[R04] Rustamov, R.: Surgery formula for the renormalized Euler characteristic of Heegaard Floer homology. arXiv:math/0409294 